Research Paper

\title{
Analysis of Clinical and Pathologic Factors of Pure, Flat Epithelial Atypia on Core Needle Biopsy to Aid in the Decision of Excision or Observation
} \author{
Erika J Schneble ${ }^{1}$, Aaron D Kirkpatrick ${ }^{5}$, Jeffrey S Saenger ${ }^{6}$, and George E Peoples ${ }^{7 凶}$ \\ 1. Department of Surgery, San Antonio Military Medical Center, Fort Sam Houston, TX; \\ 2. Department of Surgery, Madigan Military Medical Center, Fort Lewis, WA; \\ 3. Department of Surgical Oncology, University of Texas, M.D. Anderson Cancer Center, Houston, TX; \\ 4. Qwest Care Associates, Intensive Care Unit, Metropolitan Methodist Hospital, San Antonio, TX; \\ 5. Department of Radiology, San Antonio Military Medical Center, Fort Sam Houston, TX; \\ 6. Department of Pathology, San Antonio Military Medical Center, Fort Sam Houston, TX \\ 7. Director, Cancer Vaccine Development Program, San Antonio, TX, USA.
}

John S Berry11, Alfred F Trappey1, Timothy J Vreeland1, Adam R Pattyn², Guy T Clifton², Elizabeth A Berry4,

$\triangle$ Corresponding author: Dr. John S. Berry, M.D. San Antonio Military Medical Center, Department of General Surgery 3551 Roger Brooke Dr. Ft. Sam Houston, TX 78234 Office: 210-916-0439 Fax: 210-916-6658 Email: john.s.berry.mil@mail.mil.

(C) Ivyspring International Publisher. Reproduction is permitted for personal, noncommercial use, provided that the article is in whole, unmodified, and properly cited. See http://ivyspring.com/terms for terms and conditions.

Received: 2015.05.24; Accepted: 2015.08.30; Published: 2016.01.01

\begin{abstract}
Background: The optimal treatment of flat epithelial atypia (FEA) found on breast core needle biopsy (CNB) is controversial. We performed a retrospective review of our institutional experience with FEA to determine if excisional biopsy may be deferred.

Methods: Surgical records from 2009 to 2012 were reviewed for FEA diagnosis. After exclusion for concomitant lesions, CNBs of pure FEA were classified using a previously agreed upon descriptor of "focal" versus "prominent". Data was analyzed with the Fisher's Exact and Student-t test as appropriate.

Results: Of 71 CNBs evaluated, pure FEA was identified on 27 CNBs. Final excisional biopsy was benign in 24 of 27 cases (88\%) with associated ductal carcinoma in-situ (DCIS) in 3 of 27 cases (11\%). Eighteen of $27(67 \%)$ CNBs were classified as focal while $9(33 \%)$ were described as prominent. Zero of the 18 focal patients had a malignancy compared to 3 of the 9 in the prominent group ( $0 \%$ vs $33 \%, p=0.02)$. Of the 27 pure FEA CNBs, 6 patients had a personal history of breast carcinoma, five DCIS and one invasive ductal carcinoma. No malignancies were found in the 21 patients without a personal history of breast carcinoma versus three in the patients with a positive history $(0 / 21 \vee 3 / 6, p=0.007)$.

Conclusions: Our data suggests those women who have adequate sampling and sectioning of CNBs, with focal, pure FEA on pathology, and are without a personal history of breast cancer may undergo a period of imaging surveillance. Conversely, patients with a history of breast cancer or pure, prominent FEA on CNB disease should proceed to excisional biopsy.
\end{abstract}

Key words: Pure Flat Epithelial Atypia, Ductal Intraepithelial Neoplasia, Pure FEA, DIN 1A, Columnar Cell Change with atypia, Columnar Cell Hyperplasia with Atypia.

\section{Introduction}

Since its discovery in 1979 by Azzopardi, flat epithelial atypia (FEA), then called "clinging carcinoma in situ", has spurred debate as a boundary le- sion between the obvious architectural atypia of atypical ductal hyperplasia $(\mathrm{ADH})$ and the normal cytologic appearance of usual ductal hyperplasia (1). 
FEA's cytologic abnormalities are subtle and often confined to a few ducts. Today, the clinical significance of FEA as a precursor to cancer is still under discussion despite Monifar's and Simpson's isolation of genetic alterations in FEA similar to adjacent, low-grade ductal carcinoma in-situ (DCIS) and well-differentiated invasive carcinomas $(2,3)$.

The terminology describing early cytologic changes associated with breast cancer was consolidated in 2003 by the World Health Organization (WHO) and named FEA as ductal intraepithelial neoplasia (DIN) 1A (4). Synonymous terms include FEA and a B3 breast lesion which is appropriately defined as a lesion of uncertain malignant potential by the Nation Coordinating Group for Breast Screening Pathology (NHSBSP) in their B classification system. Prior to 2003, without a proper term to describe the cytologic atypia of FEA, pathologists described the entity with a wide variety of names including: columnar cell change with atypia, atypical columnar alteration with prominent apical snouts and secretions, columnar cell hyperplasia with atypia, atypical cystic duct and lobules, clinging carcinoma (monomorphic type), columnar alternation with prominent apical snouts, and more (5). Its many names confused the literature and divided opinions on the neoplastic nature of this lesion. Now, the WHO Working Group on Pathology and Genetics of Tumors of the Breast has defined FEA as "a presumably neoplastic intraductal alteration characterized by replacement of the native epithelial cells by a single or 3-5 layers of mildly atypical cells." (4) FEA's histopathology is distinguished from more benign lesions such as usual ductal hyperplasia by the presence of cytologic atypia and from more atypical lesions such as ADH (DIN1B) and low grade DCIS (DIN 1C) by the absence of architectural atypia. This consolidated definition has facilitated the understanding of FEA, but a discussion still exists regarding whether excision is warranted when pure FEA, or FEA as the most advanced lesion, is found on core needle biopsy (CNB). (6-8)

The incidence of pure FEA on CNB excluding the presence of more malignant lesions like $\mathrm{ADH}$, atypical lobular hyperplasia (ALH), lobular carcinoma in-situ (LCIS), DCIS, or invasive ductal carcinoma is between $0.72-12.2 \%$ (9-11). The underestimation (UE) rate of DCIS or invasive carcinoma after pure FEA discovered on CNB has previously been reported, ranging from $0-30 \%$ (9-12). The variability in reported UE rates could be attributed to interobserver variability in identifying pure FEA, changing definitions of FEA, the inherent subjectivity of histologic interpretation, the small amount of published data, the lack of large samples, and the long duration necessary for prospective trials. Here, we describe our 3-year singleinstitution experience with pure FEA on CNB and offer both clinical and pathologic factors which support deferred excision biopsy in favor of radiologic follow-up.

\section{Methods}

\section{Study design}

This study was part of a department-wide quality improvement initiative focused on improving breast cancer care. As part of a quality improvement project, the Department of Clinical Investigation at San Antonio Military Medical Center waived the requirement for a formal submission to the Institutional Review Board. The details of the study were reviewed by the quality improvement committee, which approved the collection of the data.

\section{Study population}

Three years of electronic medical records (January 2009 to January 2012) of active and retired military personnel and their families were queried for the terms "flat epithelial atypia", "epithelial atypia", and "breast atypia". Patients with additional pathologic designations to include invasive carcinoma, DCIS, LCIS, atypical ductal hyperplasia (ADH), atypical lobular hyperplasia (ALH), phyllodes tumor, pseudoangiomatous stromal hyperplasia (PASH), or fibroadenoma were excluded. Those patients who did not undergo an excisional biopsy, whose final pathology was not available, or whose excisional biopsy did not contain breast tissue were also excluded.

\section{Clinical Data}

The remaining records were reviewed for patient history including previous malignancies, mammographic findings, Breast Imaging and Reporting and Data System (BIRADS) classification, radiologic features, imaging guidance modality, gauge or number of biopsies, the interval between CNB and surgical excision, and pathology examination including previously agreed upon descriptors of FEA, "focal" versus "prominent", . Focal FEA was defined as FEA involving fewer than three adjacent acinar spaces within a lobule or adjacent lobules, while prominent FEA involves widespread acini with FEA and/or a larger confluent focus of FEA (Figure 1). All CNBs were processed via $3 \mathrm{H} \& \mathrm{E}-$ stained step sections; if questions remained regarding the diagnosis, additional material was obtained using previously cut, unstained material. A final review of pathologic specimens was conducted by the chief of pathology. 


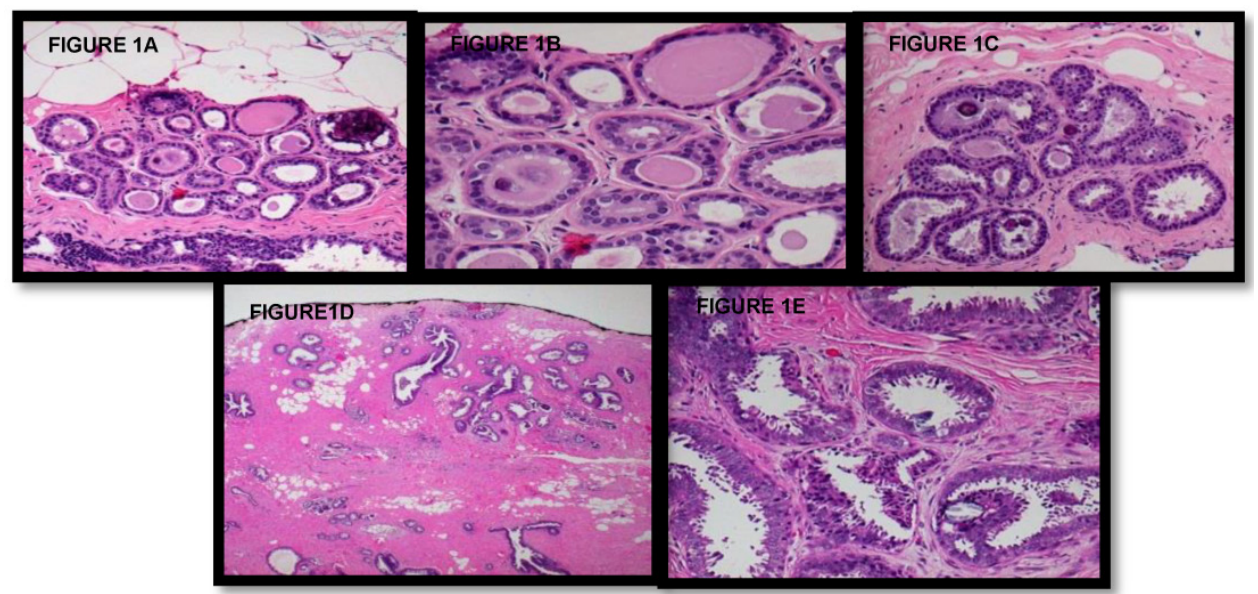

FIGURES 1: A-B Lowest end of Focal Flat Epithelial Atypia spectrum (at 10x and 20x magnification, respectively). Within this lobule, some acini exhibit round dilated acini with subtly enlarged and rounded nuclei with increased nuclear to cytoplasmic ratio (leading to basophilic appearance from hyperchromasia) and prominent nucleoli, and some nuclei no longer are oriented perpendicular to the acini's basement membrane (microscopic features of FEA). Secretions and microcalcifications are present within the some of the acinar lumina (which can be seen in FEA or non-involved acini). C Focal Flat Epithelial Atypia at 10x magnification, single lobule with the basophilic appearance characteristic of increased nuclear to cytoplasmic ratio and nuclear hyperchromasia; other nuclear atypia consisting of nucleoli and disorganized nuclear arrangement is visible along with occasional bi-nucleation. Some cells have apical snouts, a non-specific finding. D-E Prominent Flat Epithelial Atypia (10x magnification): Multiple lobules with enlarged, rounded or irregularly dilated acini, which exhibit basophilia due to increased nuclear to cytoplasmic ratio and nuclear hyperchromasia, are evident. Prominent Flat Epithelial Atypia (20x magnification): Monomorphic population of rounded and enlarged nuclei, not regularly oriented perpendicular to the basement membrane, with prominent nucleoli lining multiple lobules. Some acini contain microcalcifications and majority of cells exhibit apical snouts.

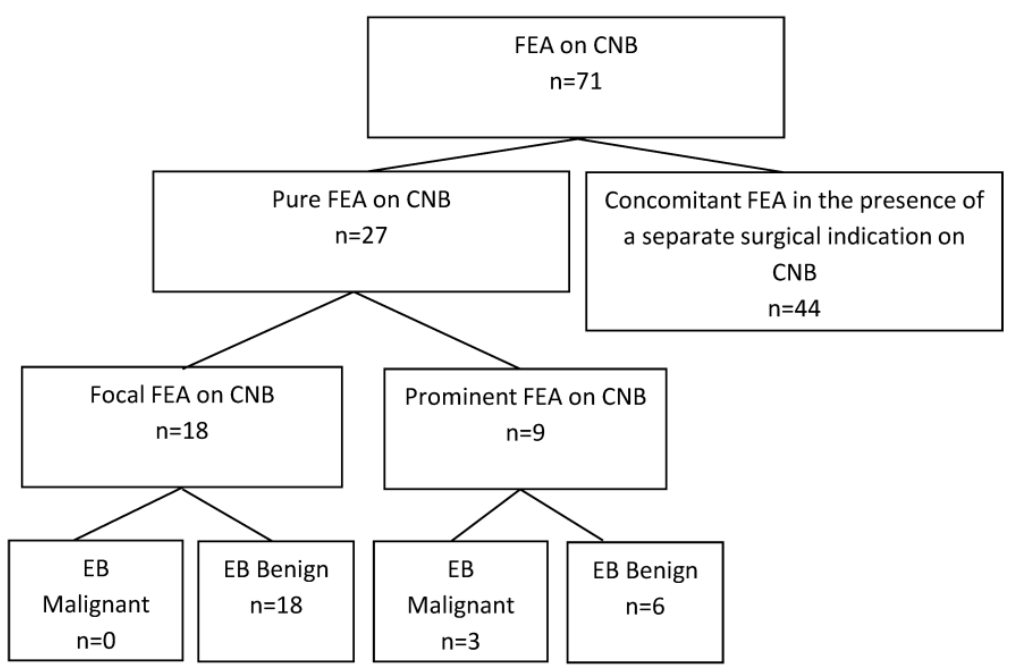

Figure 2: A total of 71 core needle biopsies (CNBs) with flat epithelial atypia (FEA) were reviewed. Sixty-two percent ( $\mathrm{n}=44$ ) were excluded for presence of a separate surgical indication or lack of adequate excisional biopsy (EB). Pure FEA core needle biopsies were designated as "focal" or "prominent". The focal FEA group contained no malignancies on excisional biopsy, compared to $33 \%(n=3)$ in the prominent group $(0 \% v .33 \%, p=0.02)$.

\section{Statistics}

Statistical analysis was based on Student's t-tests for parametric continuous variables and Fisher's exact test for comparison of categorical variables. $P$ values less than 0.05 were considered significantly different. Statistical analyses were performed using SPSS Version 19.

\section{Results}

\section{Study population}

A total of $148 \mathrm{CNBs}$ were identified from a review of electronic medical records from January 2009 to January 2012 using a query of the terms "flat epithelial atypia", "epithelial atypia", and "breast atyp- ia". Upon further review of the pathology, FEA was found to be present in $48 \%$ of these CNBs $(n=71)$. Of the 71 CNBs specimens with FEA, 55\% $(n=39)$ were excluded due to the presence of the concomitant pathologic designations described above (Figure 2). These lesions included: $21 \%(n=15)$ ADH, 16\% $(n=11)$ DCIS, $6 \%(n=4)$ LCIS, $4 \%(n=3)$ PASH, 3\% $(n=2)$ invasive carcinoma, $3 \%(\mathrm{n}=2)$ ALH, $1 \%(\mathrm{n}=1)$ fibroadenoma, and $1 \%(n=1)$ phyllodes tumor. Of the remaining $32 \mathrm{CNBs}, 7 \%(n=5)$ were also excluded due to the lack of an excisional biopsy, an excisional biopsy that did not contain breast tissue, or no excisional biopsy pathology record. As a result, pure FEA was identified from $38 \%$ of the 71 CNBs with FEA $(n=27)$ (Figure 3). 


\section{CNB Results}

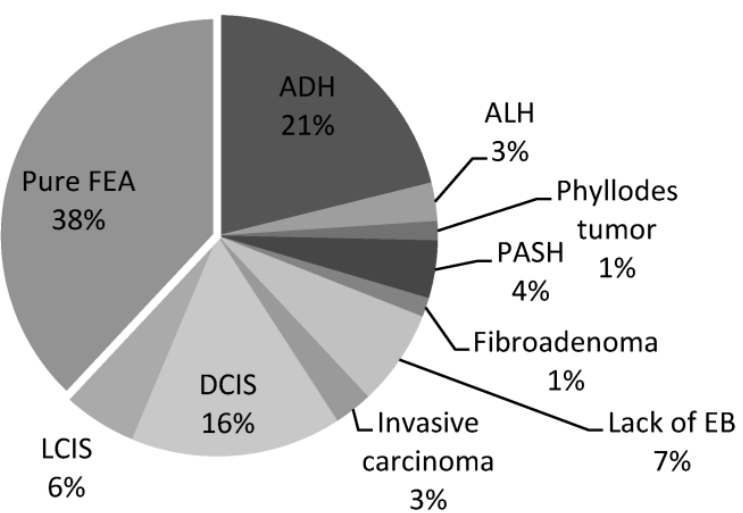

Figure 3: Pure, Flat epithelial atypia was identified on $38 \%(27 / 71)$ of core needle biopsies (CNBs). Sixty-two percent of patients were excluded for a lack of an adequate excisional biopsy or the presence of a concomitant lesion; ductal carcinoma in-situ (DCIS), lobular carcinoma in-situ (LCIS), atypical ductal hyperplasia $(A D H)$, atypical lobular hyperplasia $(A L H)$, phyllodes tumor, pseudoangiomatous stromal hyperplasia (PASH), or fibroadenoma.

\section{Pure FEA}

Of the 71 CNBs with FEA, 27 contained pure FEA and will be the focus of this analysis. CNBs of pure FEA were largely the result of a BIRADS 4 or greater designation $(92 \%, \mathrm{n}=25)$. Specifically, suspicious microcalcifications accounted for $67 \%$ of pure FEA CNBs $(n=18)$ and the remaining $33 \%(n=9)$ of CNBs were done for focal asymmetry. Stereotactic guidance was performed for $67 \%(n=18)$ of pure FEA CNBs. The remaining CNBs were guided by ultrasound, $30 \%(\mathrm{n}=8)$, or by magnetic resonance imaging (MRI), 4\% $(n=1)$. Seventy-four percent $(n=20)$ of CNBs were done with an 8-gauge needle with a mean of $5.3+1.3$ biopsies (Table 1). All patients underwent a needle localized, excisional breast biopsy performed by eight different surgeons. Mean time between CNB and excisional biopsy was 37 days, range 7-261.

\section{Focal vs. prominent FEA}

Pure FEA was identified from 27 patients. After CNB, $67 \%(n=18)$ had a single focus of FEA while $33 \%$ $(n=9)$ were described as prominent. There were no statistical differences between the focal and prominent FEA groups with respect to mammographic findings worthy of biopsy including microcalcifications, BIRADS classification, imaging guidance modality, gauge or number of biopsies, laterality of previous cancers, or the interval between $\mathrm{CNB}$ and surgical excision groups (Table 1). After excisional biopsy, no malignancies $(0 / 18)$ were identified in the focal, pure FEA group compared to $33 \%(n=3 / 9)$ of the prominent group $(0 \%$ v. $33 \%, p=0.02)$ (Figure 2$)$.
Table 1: Demographics of those patients with a CNB identified as pure FEA.

\begin{tabular}{|c|c|c|c|}
\hline \multicolumn{4}{|c|}{ Demographics } \\
\hline & Focal $(n=18)$ & Prominent $(n=9)$ & $\mathrm{p}$ \\
\hline Age & 51 & 55 & 0.33 \\
\hline \multicolumn{4}{|l|}{ Number of Biopsies } \\
\hline 4 & $28 \%$ & $22 \%$ & 1.00 \\
\hline 5 & $11 \%$ & $22 \%$ & 0.58 \\
\hline 6 & $39 \%$ & $44 \%$ & 1.00 \\
\hline 7 & $11 \%$ & $0 \%$ & 0.54 \\
\hline 8 & $11 \%$ & $11 \%$ & 1.00 \\
\hline \multicolumn{4}{|l|}{ Size of Biopsy } \\
\hline 8 gauge & $83 \%$ & $56 \%$ & 0.18 \\
\hline 11 gauge & $11 \%$ & $22 \%$ & 0.58 \\
\hline 14 gauge & $6 \%$ & $22 \%$ & 0.25 \\
\hline \multicolumn{4}{|l|}{ Indication for Biopsy } \\
\hline Suspicious Microcalcifications & $72 \%$ & $44 \%$ & 0.22 \\
\hline Mammographic Asymetry & $28 \%$ & $44 \%$ & 0.42 \\
\hline \multicolumn{4}{|l|}{ Biopsy Guidance } \\
\hline Stereotactic & $72 \%$ & $56 \%$ & 0.42 \\
\hline Ultrasound & $22 \%$ & $44 \%$ & 0.38 \\
\hline MRI & $6 \%$ & $0 \%$ & 1.00 \\
\hline \multicolumn{4}{|l|}{ BIRADS score } \\
\hline BIRADS 3 & $6 \%$ & $11 \%$ & 1.00 \\
\hline BIRADS 4 & $89 \%$ & $89 \%$ & 1.00 \\
\hline History of Breast Cancer & $6 \%$ & $56 \%$ & 0.01 \\
\hline Malignancy on Surgical Biopsy & $0 \%$ & $33 \%$ & 0.03 \\
\hline
\end{tabular}

\section{History of Carcinoma}

The 27 pure, FEA biopsies findings were subdivided into focal FEA in $(66.7 \%, \mathrm{n}=18)$ and prominent FEA $(33.3 \%, n=9)$. After surgical excision, no cases of invasive carcinoma were identified from the $27 \mathrm{CNBs}$ identified as pure FEA. Benign findings occurred in $88.8 \%(n=24)$ of the pure FEA excisional biopsies and DCIS was reported in the remaining $11.2 \%(n=3)$. Of the 27 pure FEA CNBs, $22 \%(n=6)$ patients had a personal history of breast cancer: $19 \%(n=5)$ DCIS and 3\% $(n=1)$ invasive ductal carcinoma. Of the 6 patients with prior breast cancer, $1 \mathrm{CNB}$ was classified as focal FEA while 5 CNBs were prominent FEA. Four of the pure FEA biopsies were in the ipsilateral breast, while two were in the contralateral breast $(p=0.33)$. There were no malignancies found in the 21 patients without a personal history of breast carcinoma versus $50 \%$ $(n=3)$ in the patients with a history of breast cancer (0/21 v. 3/6, p=0.007).

\section{Discussion}

The rate at which CNB fails to predict more malignant lesions on surgical excision is termed the underestimation (UE) rate. As expected, the UE rate is a function of the amount of tissue sampled at CNB, sampling accuracy of the imaged abnormality, and the slide rating defined by the number of slides through a surgical specimen. In our study, the UE rate of carcinoma for a CNB of pure FEA was $11 \%$. If focal FEA was adequately demonstrated with $\mathrm{CNB}$, the 
pure FEA UE rate was $0 \%$ compared to a $33 \%$ UE rate in prominent FEA. In regards to clinical characteristics, a personal history of breast cancer combined with pure FEA on CNB resulted in an UE rate of $50 \%$.

To determine the incidence of FEA as a marker lesion of adjacent cancer, a retrospective review of excision, core, or mastectomy specimens with microcalcifications was undertaken by De Mascarel et al. (13). After reviewing 971 surgically excised biopsies with atypia, pure FEA with concomitant cancer was identified in $17 \%$ of cases. In a smaller sample size, Abdel-Fatah et al. show a FEA, not pure FEA, to tubular carcinoma association as high as $84 \%$ in 56 patients with a lesser association with invasive lobular carcinoma of $54 \%$ in 57 patients (14). Therefore the true incidence of concomitant pure FEA and breast cancer is hard to predict but is probably close to $17 \%$. However, these studies do not evaluate the typical clinical management pathway for evaluating suspicious mammographic lesions, which might include image guided $\mathrm{CNB}$, surgical excision, or follow-up imaging and instead evaluate only the surgical biopsy results.

In comparison studies, using the typical clinical pathway, incidence of isolated pure FEA on CNB is considerably lower, between $0.72-12.2 \%$ (9-11). The difference between these two rates, the $17 \%$ incidence of pure FEA with cancer at surgical specimen and $0.72-12.2 \%$ pure FEA on CNB and associated cancer on surgical excision, is likely the true UE rate. In the current literature, if the FEA on CNB is used to predict excisional biopsy results, UE rates regardless of CNB gauge are between $0-29 \%$ (7-12, 15-19). In studies using a 14-gauge $\mathrm{CNB}$, UE rates are between $14-21 \%$ with an average number of 5 samples or $200 \mathrm{mg}$ of tissue $(10,17)$. In studies with 11-gauge CNB, UE rates are between $0-20 \%(7,9,12,19-20)$. In this study, $74 \%$ of CNBs of pure FEA were performed with 8-gauge core needles, and the mean number of CNBs was 6 (approximately $600 \mathrm{mg}$ of tissue). The UE rate for all isolated FEA on CNB in our institutional experience was found to be $11 \%$, similar to previous studies using 11-gauge sampling, and a rate at which many surgeons would feel uncomfortable with radiographic surveillance alone.

Identifying subsets of pure FEA with a lower risk of UE, who therefore could safely be observed with serial imaging alone, could decrease the time, cost, discomfort and morbidity of excisional biopsy in many cases. The idea to subdivide FEA was undertaken by Bonnett et al. (20) in a retrospective analysis of atypical lesions in image guided core biopsies. The investigators found that after categorizing ALH, ADH with minimal atypia, $\mathrm{ADH} /$ borderline DCIS, and FEA by the number of foci on $\mathrm{CNB}$, the number of involved foci correlated to the presence of DCIS on open biopsy (20). In a different study, Martel et al. (10) reviewed 1,751 CNBs over eight years, 3.6\% $(n=63)$ of which were FEA as the most advanced pathology, not pure FEA. Cases were further characterized by the extent of FEA; specifically into those having less than five terminal ductal lobular units (TDLUs), five to ten TDLUs, or greater than 10 TDLUs involved. Of the seven patients with FEA and ipsilateral invasive carcinoma, $5(71 \%)$ had greater than 10 ductules (greater than 2 terminal duct lobular units TDLUs) involved (10). Of note, the mean interval between initial CNB and surgical excision for this study was 3.7 years.

Pathologists at San Antonio Military Medical Center began prospectively labeling cases of pure FEA as focal or prominent beginning in 2007. At our three year review, the UE rate in those with prominent FEA is $33 \%$ while the UE rate in those with focal FEA is $0 \%$. Keeping with the previous findings of Bonnet et al. (20), this difference in UE rates suggests prominent FEA may better identify patients at risk for surrounding DCIS or invasive carcinoma and thus guide surgical excision. While focal FEA may become prominent over time, it poses little risk of surrounding cancer at the time of CNB. Further prospective studies will be needed to determine the longer-term risk of transformation into more aggressive lesions. However, in a recent long-term cohort study from the Mayo Clinic in 11,591 women with benign excisional breast biopsies with a median follow-up of 16.8 years, the finding of FEA did not appear to impart independent risk of breast cancer beyond that atypical hyperplasia or proliferative disease without atypia (21).

After demographic analysis, no malignancies were found in the 21 patients without a personal history of invasive carcinoma or DCIS versus 3 in the 6 patients with a history of carcinoma. In addition, there were significantly more women with a personal history of breast cancer in the prominent, pure FEA group (5) than in the focal, pure FEA group (1). Therefore, the effect of a personal history of breast cancer can be difficult to separate from the pathologic designation (prominent vs. focal) of FEA. The role of personal history of breast cancer in FEA UE rate is mixed in previous studies. A retrospective analysis by Peres et al. (19) found no correlation between personal history of cancer and underestimation of carcinoma in 128 cases of pure FEA by 11 gauge CNB obtained over a 7-year period. In contrast, Lavoue and Becker et al. $(8,11)$ demonstrate that $18-30 \%$ of women who were underestimated by pure FEA on CNB had a personal or family history of breast cancer. Taken as a whole, until better data is available, it is prudent to consider personal history when deciding not to proceed with 
excisional biopsy based on a CNB finding of pure FEA.

Our study is also limited by the relatively small number of cases of pure FEA within CNBs; however, our series represents one of the largest studies of pure FEA and is unique in the attempt to divide FEA into histologic subgroups.

\section{Conclusion}

It is unlikely that any single factor will be sufficient to determine whether a patient with pure FEA on CNB should forgo excisional biopsy. Our data suggests those women with a history of breast cancer or pure, prominent FEA on CNB should proceed to excisional biopsy. Conversely, those patients who have adequate sampling and sectioning of CNBs, with focal, pure FEA on pathology, and are without a personal history of breast cancer may undergo a period of imaging surveillance.

\section{Acknowledgements}

The authors would like to thank Mrs. Susie Ferrise and Mrs. Karen Degroat for their database management, editorial expertise, and literary searches.

\section{Abbreviations}

FEA: Focal epithelial atypia; CNB: core needle biopsy; EB: excisional biopsy; PASH: pseudoangiomatous stromal hyperplasia; $\mathrm{ADH}$ : atypical ductal hyperplasia; ALH: atypical lobular hyperplasia; LCIS: lobular carcinoma in-situ; DCIS: ductal carcinoma in-situ; DIN: ductal intraepithelial neoplasia; WHO: World Health Organization; BIRADS: Breast Imaging and Reporting and Data System; TDLU: terminal ductal lobular unit.

\section{Competing Interests}

The authors have declared that no competing interest exists.

\section{References}

1. Azzopardi JG, Ahmed A, Millis RR. Problems in breast pathology. Major Problems in Breast Pathology. 1979; 11: i- xvi, 1-466.

2. Moinfar F, Man YG, Bratthauer GL, Ratschek M, Tavassoli FA. Genetic abnormalities in mammary ductal intraepithelial neoplasia-flat type ("clinging ductal carcinoma in situ"): a simulator of normal mammary epithelium. Cancer 2000; 88: 2072-2081.

3. Simpson PT, Gale T, Reis-Filho JS, et al. Columnar cell lesions of the breast: the missing link in breast cancer progression? A morphological and molecular analysis. Am J Surg Pathol 2005;29:734-746.

4. Tavassoli FA HH, Rosai J, Holland R, Ellis IO, Schnitt SJ, Boecker W, Heywang Kobrunner SH, Moinfar F, Lakhani SR. Intraductal proliferative lesions. In: Tavassoli FA DP, ed. Pathology and Genetics of Tumours of the Breast and Female Genital Organs. Lyons, France: IARC Press; 2003: 63-73.

5. Schnitt SJ, Vincent-Salomon A. Columnar cell lesions of the breast. Adv Anat Pathol. 2003; 10: 113-124.

6. Lieske B, Ravichandran D, Alvi A, Lawrence DA, Wright DJ. Screen-detected breast lesions with an indeterminate (B3) core needle biopsy should be excised. Eur J Surg Oncol 2008; 34: 1293- 1298.

7. Yamaguchi R, Tanaka M, Tse GM, et al. Pure flat epithelial atypia is uncommon in subsequent breast excisions for atypical epithelial proliferation. Cancer Sci 2012; 103: 1580-1585.
8. Lavoue V, Roger CM, Poilblanc M, et al. Pure flat epithelial atypia (DIN 1a) on core needle biopsy: study of 60 biopsies with follow-up surgical excision. Breast Cancer Res Treat 2011; 125: 121-126.

9. Piubello Q, Parisi A, Eccher A, Barbazeni G, Franchini Z, Iannucci A. Flat epithelial atypia on core needle biopsy: which is the right management? Am J Surg Pathol 2009; 33: 1078-1084.

10. Martel M, Barron-Rodriguez P, Tolgay Ocal I, Dotto J, Tavassoli FA. Flat DIN 1 (flat epithelial atypia) on core needle biopsy: 63 cases identified retrospectively among 1,751 core biopsies performed over an 8-year period (1992-1999). Virchows Arch 2007; 451: 883-891.

11. Becker AK, Gordon PB, Harrison DA, et al. Flat ductal intraepithelial neoplasia 1A diagnosed at stereotactic core needle biopsy: is excisional biopsy indicated? AJR Am J Roentgenol 2013; 200: 682-688.

12. Senetta R, Campanino PP, Mariscotti G, et al. Columnar cell lesions associated with breast calcifications on vacuum-assisted core biopsies: clinical, radiographic, and histological correlations. Mod Pathol 2009; 22: 762-769.

13. de Mascarel I, MacGrogan G, Mathoulin-Pelissier S, et al. Epithelial atypia in biopsies performed for microcalcifications. practical considerations about 2,833 serially sectioned surgical biopsies with a long follow-up. Virchows Arch 2007;451:1-10.

14. Abdel-Fatah TM, Powe DG, Hodi Z, et al. High frequency of coexistence of columnar cell lesions, lobular neoplasia, and low grade ductal carcinoma with invasive tubular carcinoma and invasive lobular carcinoma. Am J Surg Pathol 2007; 31: 417-426.

15. Noel JC, Buxant F, Engohan-Aloghe C. Immediate surgical resection of residual microcalcifications after a diagnosis of pure flat epithelial atypia on core biopsy: a word of caution. Surg Oncol 2010; 19: 243-246.

16. Ingegnoli A, d'Aloia C, Frattaruolo A, et al. Flat epithelial atypia and atypical ductal hyperplasia: carcinoma underestimation rate. Breast J 2010; 16: 55-59.

17. Kunju LP, Kleer CG. Significance of flat epithelial atypia on mammotome core needle biopsy: Should it be excised? Hum Pathol 2007; 38: 35-41.

18. Lee TY, Macintosh RF, Rayson D, Barnes PJ. Flat epithelial atypia on breast needle core biopsy: a retrospective study with clinical-pathological correlation. Breast J 2010; 16: 377-383.

19. Peres A, Barranger E, Becette V, Boudinet A, Guinebretiere JM, Cherel P. Rates of upgrade to malignancy for 271 cases of flat epithelial atypia (FEA) diagnosed by breast core biopsy. Breast Cancer Res Treat 2012; 133: 659-666.

20. Bonnett M, Wallis T, Rossmann M, et al. Histopathologic analysis of atypical lesions in image- guided core breast biopsies. Mod Pathol 2003; 16: 154-160

21. Said SM, Visscher DW, Nassar A, Frank RD, Vierkant RA, Frost MH, Ghosh K, Radisky DC, Hartmann LC, Degnim AC. Flat epithelial atypia and risk of breast cancer: A Mayo cohort study. Cancer 2015; 121(10): 1548-55. 